\title{
Radio electric tissue optimization in the treatment of surgical wounds
}

This article was published in the following Dove Press journal:

Clinical, Cosmetic and Investigational Dermatology

24 August 2011

Number of times this article has been viewed

\section{Alessandro Castagna' \\ Vania Fontani ${ }^{1}$ \\ Salvatore Rinaldi ${ }^{1,2}$ \\ Piero Mannu'}

'Rinaldi Fontani Institute, ${ }^{2}$ Medical School of Occupational Medicine, University of Florence, Florence, Italy
Correspondence: Salvatore Rinaldi Rinaldi Fontani Institute, Viale Belfiore 43, $50 \mid 44$ Florence, Italy

Tel +3955 290307

Fax +3955290399

Email srinaldi@irf.it
Purpose: To report preliminary results with the tissue optimization (TO) treatment with a radio electric asymmetric conveyer (REAC) in promoting the repair of surgical wounds.

Patients and methods: Two subjects, a 54-year-old male with a tear bruise on the upper third of the leg and a 19-year-old female with a stab wound to the hand, were treated with 12 REAC-TO treatment sessions.

Results: In both patients, the wounds showed shorter healing time compared with the time usually required for similar wounds, and good repair quality.

Conclusion: REAC device with its specific treatment protocols may be an alternative therapy for wound healing.

Keywords: healing time, quality of repair, tissue regeneration, tissue repair

\section{Introduction}

Among the methods used to treat wounds ${ }^{1,2}$ are electrical stimulation ${ }^{3-5}$ and magnetic fields. ${ }^{6,7}$ More recently, a new therapeutic device, tissue optimization (TO) with a radio electric asymmetric conveyer (REAC), was developed for wound treatment. We describe the use of this device in two patients.

\section{Material and methods REAC}

The REAC (Convogliatore di Radianza Modulante, Asmed, Florence, Italy) uses patented technology (EP20010960475 20010706, US 7333859) based on innovative biostimulation technology and has been used for noninvasive brain stimulation. REAC treatments have proven efficacy in ameliorating stress-related disorders, ${ }^{8-12}$ depression, ${ }^{13}$ anxiety, ${ }^{14}$ and other psychiatric disorders, ${ }^{15,16}$ motor behavior abnormalities, ${ }^{17-19}$ and tissue stimulation. ${ }^{20}$

\section{REAC-TO}

The REAC-TO protocol consisted of 100 radio frequency bursts, each of $2.4 \mathrm{GHz}$ for 0.5 seconds, with a specific absorption rate of $7 \mu \mathrm{W} / \mathrm{kg}$, spaced with 4.5 -second pauses, applied to the skin by a laminar aluminum probe (Figure 1). Each therapy session lasted about 10 minutes, with 12 to 18 sessions constituting a REAC-TO treatment cycle. REAC-TO has demonstrated effectiveness in improving circulation, hydration, and the tropism of facial skin in healthy subjects. ${ }^{20}$ Moreover, REAC was found to have the ability to modulate the expression of genes and proteins involved 
in the differentiation of embryonic mouse stem cells and to improve tissue regeneration.

\section{Patient I}

A 54-year-old man sustained an accidental laceration to his upper right leg. The wound was sutured immediately and 10 days later he began REAC-TO treatment (Figure 2) without any other local therapy. After six REAC-TO treatments, the stitches were removed and the wound was washed with a mild solution of sodium electrolytes (Figure 3). He was treated for a total of 12 sessions (Figure 4). The wound was examined after 30 days (Figure 5) and after 2 months (Figure 6).

\section{Patient 2}

A 19-year-old woman, on estrogen-progestin therapy, sustained an accidental stab wound to the palm of her left hand, with partial lesion of the superficial flexor tendon of the fourth and fifth finger. The wounds were sutured and Zimmer frames were applied, positioned in flexion for 25 days. She received amoxicillin trihydrate orally for 7 days, pain medication as needed with ketoprofen, along with rest for 15 days. After 9 days, a few stitches were removed and 4 days later, all the remaining stitches were removed; wound dehiscence was observed, so polypropylene patches were applied. Examination 10 days later showed persistence of

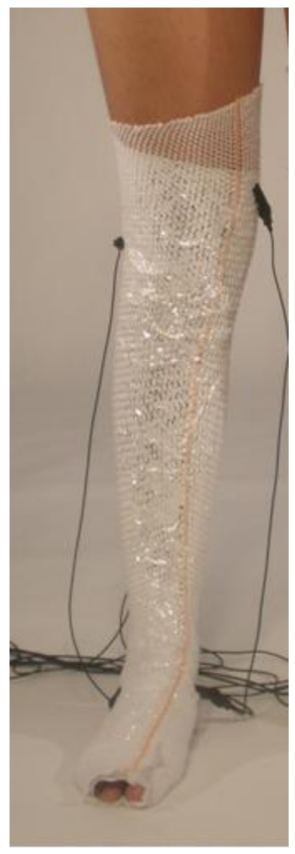

Figure I The radio electric asymmetric conveyer-tissue optimization protocol consisted of 100 radio frequency bursts, each of $2.4 \mathrm{GHz}$ for 0.5 seconds, with a specific absorption rate of $7 \mu \mathrm{W} / \mathrm{kg}$, spaced with 4.5 second pauses, applied to the skin by a laminar aluminum probe.

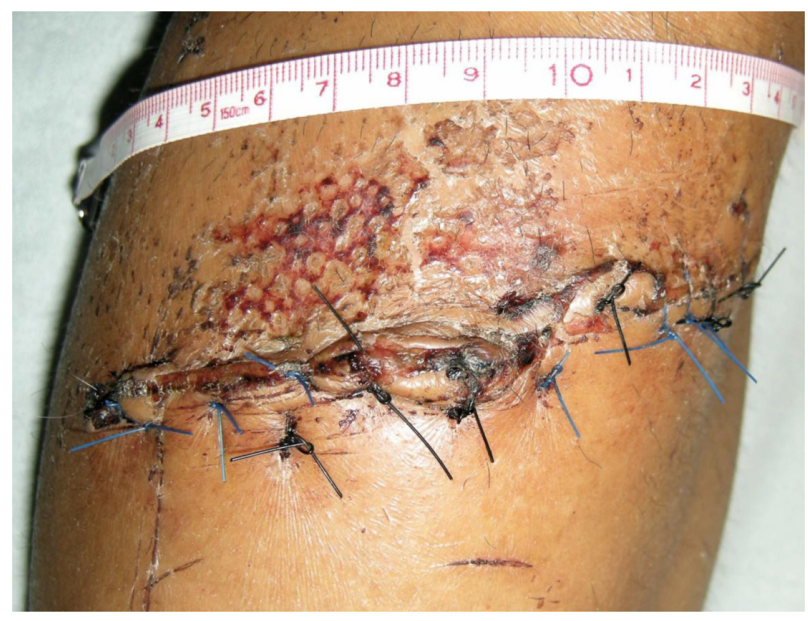

Figure 2 Case I at T0, before radio electric asymmetric conveyer-tissue optimization treatment.

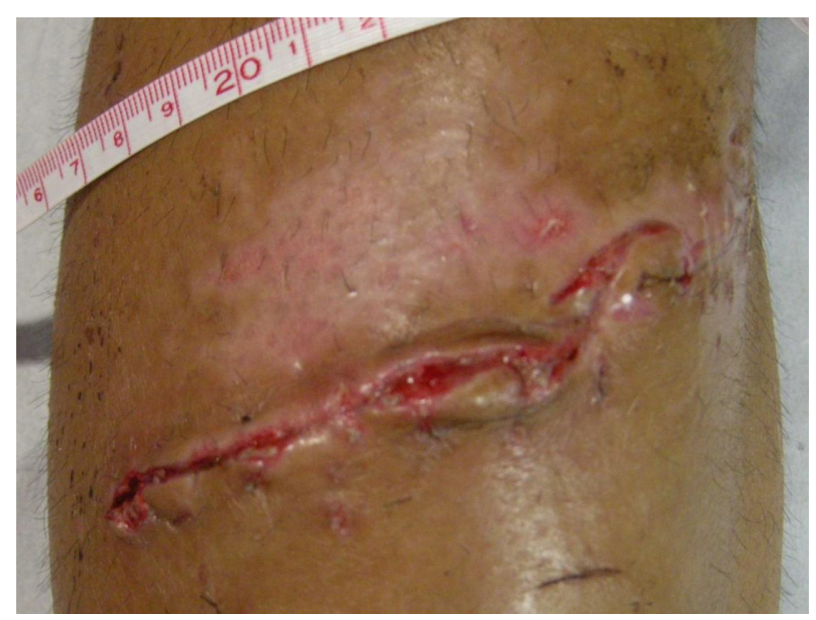

Figure 3 Case I at TI, after six radio electric asymmetric conveyer-tissue optimization treatments.

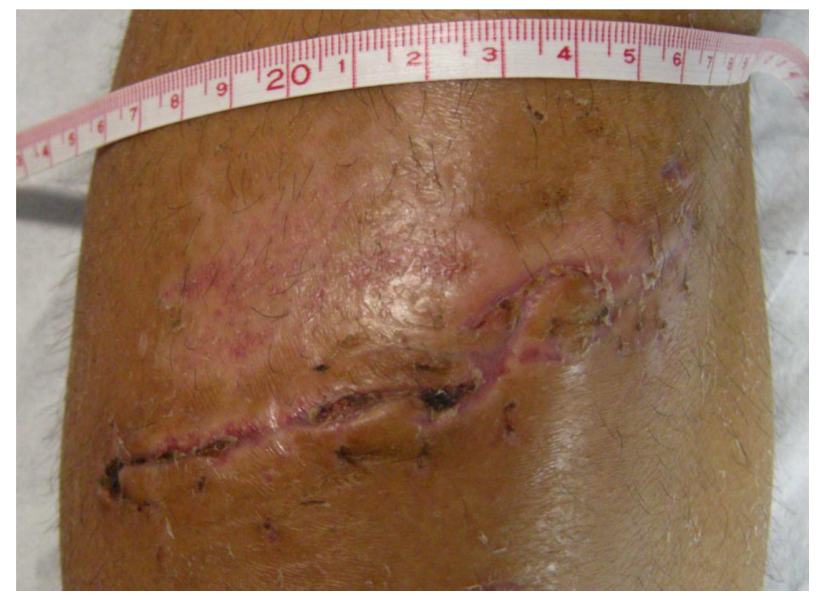

Figure 4 Case 1 at T2, after 12 radio electric asymmetric conveyer-tissue optimization treatments. 


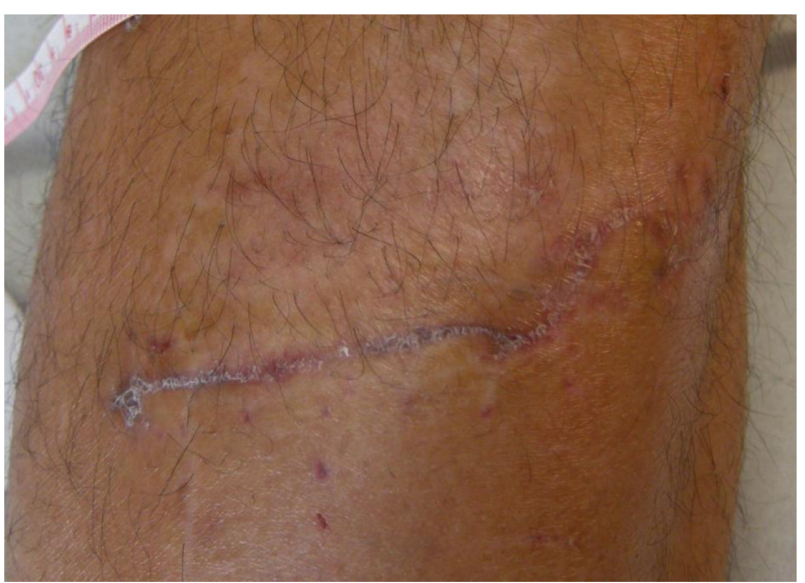

Figure 5 Case I at T3, after 30 days.

wound dehiscence (Figure 7). After washing and disinfecting the wound, we began treating with REAC-TO, applied to the palm of her left hand. Treatments were applied twice daily, once each in the morning and evening, for a total of 12 sessions (Figures 8-11). During REAC-TO, curettages and local medical treatment were not applied, apart from disinfection with a spray containing a mild solution of sodium electrolytes and the application of a hydro-absorbent multi-layer gauze, maintained by noncompressive elastic tubular gauze.

\section{Results}

The images of the two patients clearly show that the quality of repair was good and the times required were shorter than those normally observed.

\section{Discussion}

Surgical wound dehiscence is problematic with regard to the quality of repair and to the timing of healing. In fact,

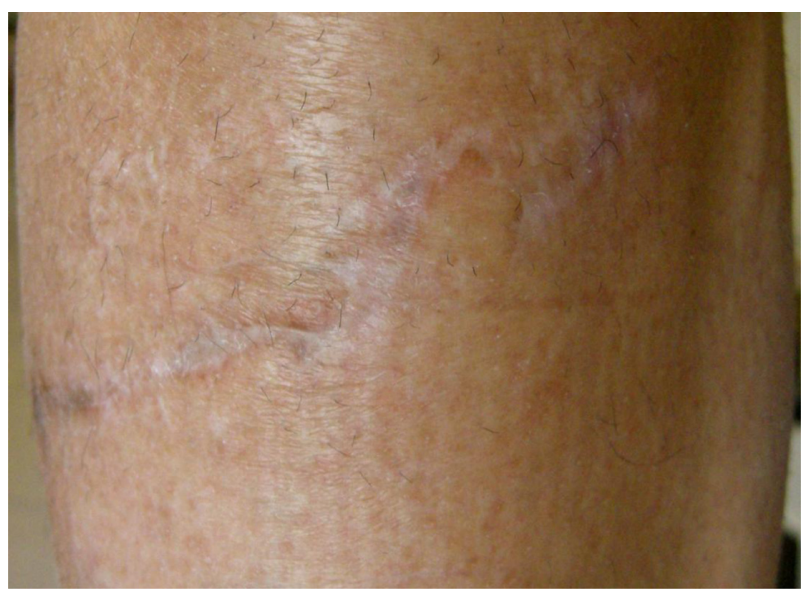

Figure 6 Case I at T4, after 60 days.

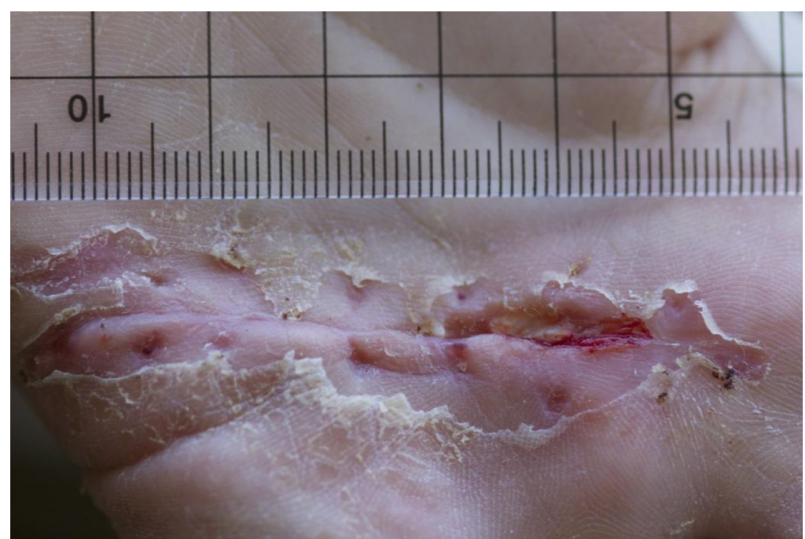

A

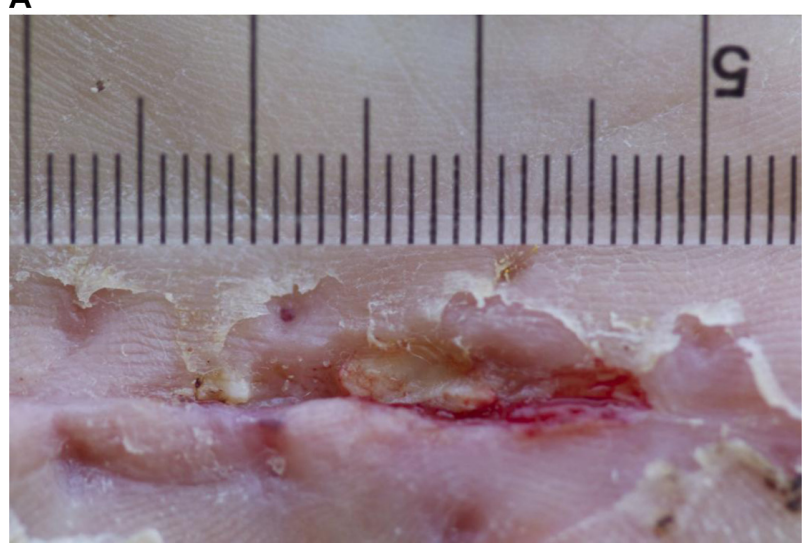

Figure 7 Case 2 at T0 and (A) detail of the image.

it is not uncommon to see retracting scars, keloids, and blemishes, not due to regeneration, but to replacement with connective tissue and then more or less evident fibrosis. On this basis, the timing of recovery is extremely varied and depends on the presence or absence of an infection, the depth of the wound, and the extension. Skin thickness and

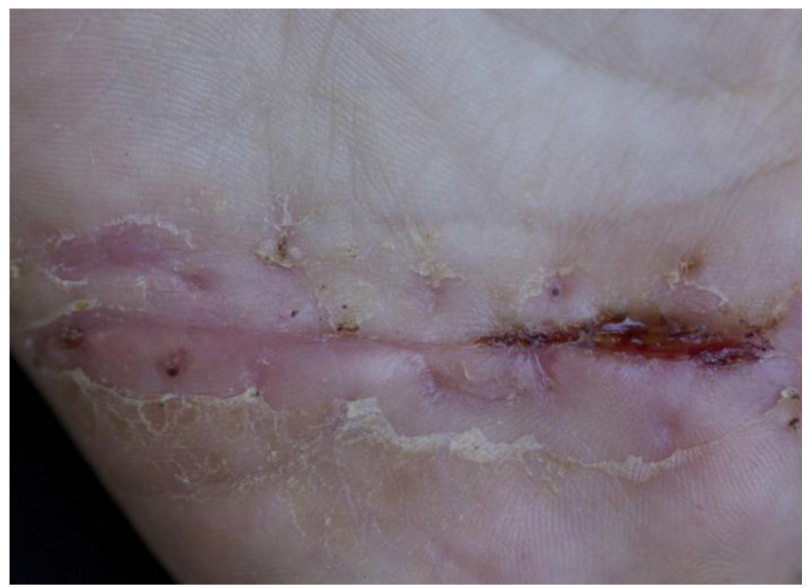

Figure 8 Case 2 at TI, after 2 days and four radio electric asymmetric conveyertissue optimization treatments. 


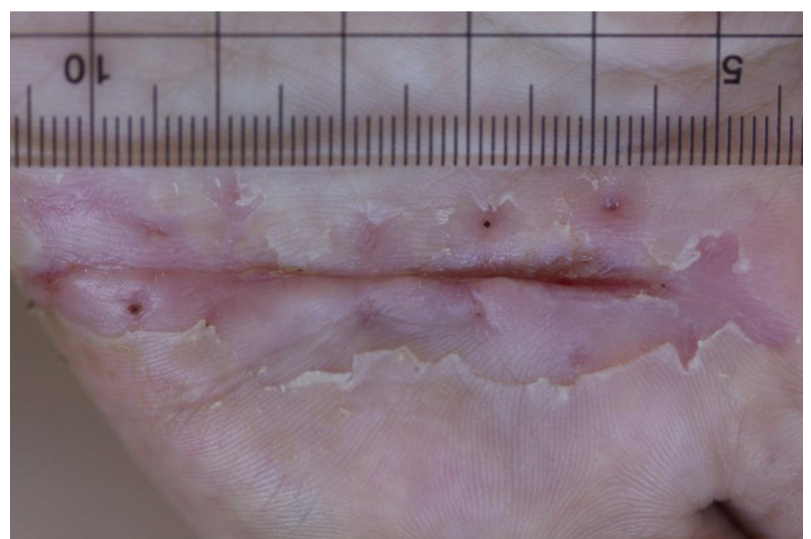

Figure 9 Case 2 at T2, after 4 days and eight radio electric asymmetric conveyertissue optimization treatments.

sweat both affect the healing process. In our experience, the healing of a wound similar to that in patient 1 would need at least 15 days and the esthetic and functional result is never predictable. The wound in question closed with impressive speed and was almost healed on day 6, with a progression that involved filling in each portion of the wound from the bottom and the margins, which is a sign of fine repair. In patient 2 we can apply the same considerations as case one. It should be noted that a favorable esthetic result was obtained, with the total absence of scarring. Although the use of REAC-TO in only two patients may be regarded as anecdotal and further studies need to be conducted, we found that REAC-TO accelerated the healing time and repair of both wounds, compared with the time usually required for similar wounds. Furthermore, this treatment was completely noninvasive, painless, and safe, and is popular among patients.

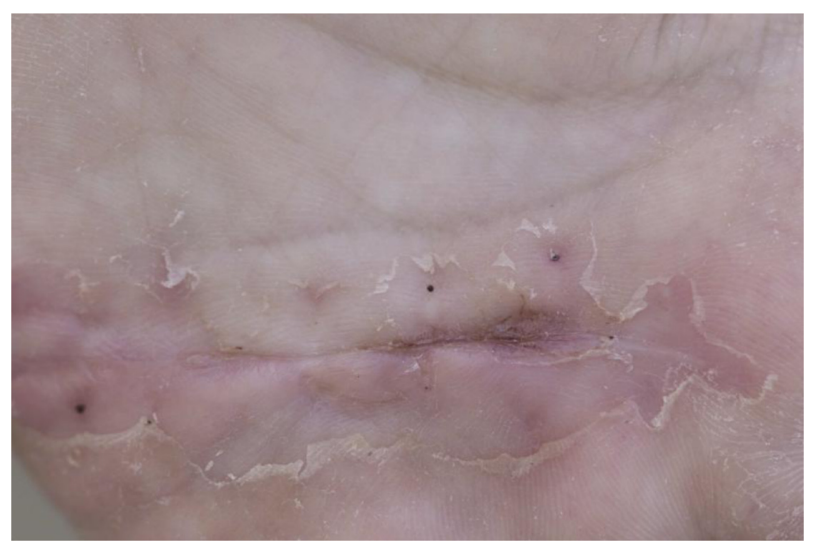

Figure 10 Case 2 at T3, after 6 days and 10 radio electric asymmetric conveyertissue optimization treatments.

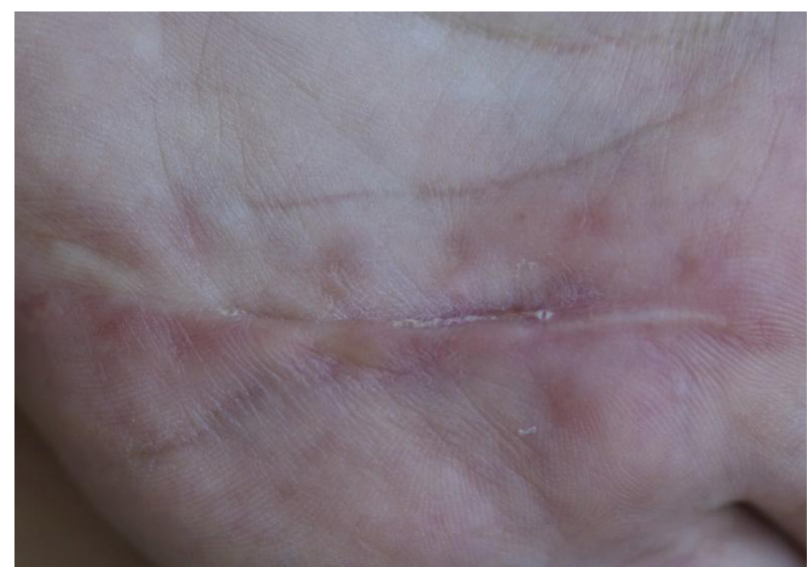

Figure I I Case 2 at T4, after 8 days and 12 radio electric asymmetric conveyertissue optimization treatments.

\section{Conclusion}

Our previous positive experiences with in vivo ${ }^{20}$ and in vitro regeneration, and the extensive experience in the use of electric stimulation and electromagnetic fields, suggest that REAC-TO may be effective in curing wounds and sores and in anti-aging treatments.

\section{Disclosure}

$\mathrm{SR}$ and $\mathrm{VF}$ are the inventors of the radio electric asymmetric conveyor.

\section{References}

1. Hess CL, Howard MA, Attinger CE. A review of mechanical adjuncts in wound healing: hydrotherapy, ultrasound, negative pressure therapy, hyperbaric oxygen, and electrostimulation. Ann Plast Surg. 2003;51(2):210-218.

2. Cullum N, Nelson EA, Flemming K, Sheldon T. Systematic reviews of wound care management: (5) beds; (6) compression; (7) laser therapy, therapeutic ultrasound, electrotherapy and electromagnetic therapy. Health Technol Assess. 2001;5(9):1-221.

3. Bullock AJ, Barker AT, Coulton L, Macneil S. The effect of induced biphasic pulsed currents on re-epithelialization of a novel wound healing model. Bioelectromagnetics. 2007;28(1):31-41.

4. Lauchli S. Alternative methods for wound treatment. MMW Fortschr Med. 2007;149(46):41-42. German.

5. Cinar K, Comlekci S, Senol N. Effects of a specially pulsed electric field on an animal model of wound healing. Lasers Med Sci. 2009;24(5):735-740.

6. Aziz Z, Cullum NA, Flemming K. Electromagnetic therapy for treating venous leg ulcers. Cochrane Database Syst Rev. 2011;3:CD002933.

7. Ravaghi H, Flemming K, Cullum N, Olyaee Manesh A. Electromagnetic therapy for treating venous leg ulcers. Cochrane Database Syst Rev. 2006;2:CD002933.

8. Rinaldi S, Fontani V, Aravagli L, Margotti ML. Psychological and symptomatic stress-related disorders with radio-electric treatment: psychometric evaluation. Stress Health. 2010;26(5):350-358.

9. Rinaldi S, Fontani V, Aravagli L, Mannu P. Psychometric evaluation of a radio electric auricular treatment for stress related disorders: a double-blinded, placebo-controlled controlled pilot study. Health Qual Life Outcomes. 2010;8:31. 
10. Rinaldi S, Fontani V, Aravagli L, et al. Stress-related psycho-physiological disorders: randomized single blind placebo controlled naturalistic study of psychometric evaluation using a radio electric asymmetric treatment. Health Qual Life Outcomes. 2011;9(1):54.

11. Rinaldi S, Fontani V, Moretti E, et al. A new approach on stressrelated depression and anxiety: Neuro-Psycho-Physical-Optimization with Radio Electric Asymmetric-Conveyer. Indian J Med Res. 2010;132:189-194.

12. Collodel G, Moretti E, Fontani V, et al. Effect of emotional stress on sperm quality. Indian J Med Res. 2008;128(3):254-261.

13. Mannu P, Rinaldi S, Fontani V, Castagna A, Margotti ML. Radio electric treatment vs escitalopram in the treatment of panic disorders associated with major depression: an open-label, naturalistic study. Acupunct Electrother Res. 2009;34(3-4):135-149.

14. Bourget Olivieri E, Vecchiato C, Ignaccolo N, et al. Radio electric brain stimulation in the treatment of generalized anxiety disorder comorbid with major depression in a public psychiatric hospital: a pilot study. Neuropsychiatr Dis Treat. 2011. In press.

15. Mannu P, Rinaldi S, Fontani V, Castagna A. Long-term treatment of bipolar disorder with a radioelectric asymmetric conveyor. Neuropsychiatr Dis Treat. 2011;7(1):373-379.
16. Mannu P, Rinaldi S, Fontani V, Castagna A. Radio electric asymmetric brain stimulation in the treatment of behavioral and psychiatric symptoms in Alzheimer Disease. Clin Interv Aging. 2011;6:207-211.

17. Castagna A, Rinaldi S, Fontani V, Aravagli L, Mannu P, Margotti ML. Does osteoarthritis of the knee also have a psychogenic component? Psycho-emotional treatment with a radio-electric device vs intraarticular injection of sodium hyaluronate: an open-label, naturalistic study. Acupunct Electrotherapeutics Res. 2010;35(1-2):1-16.

18. Castagna A, Rinaldi S, Fontani V, Mannu P, Margotti ML. Comparison of two treatments for coxarthrosis: local hyperthermia versus radio electric asymmetrical brain stimulation. Clin Interv Aging. 2011;6:201-206.

19. Castagna A, Rinaldi S, Fontani V, Mannu P. Radioelectric asymmetric brain stimulation and lingual apex repositioning in patients with atypical deglutition. J Multidiscip Healthc. 2011;4(1):209-213.

20. Rinaldi S, Fontani V, Cupelli V, et al. [Capillaroscopy changes of the face, induced by activation with REAC tissue stimulation, in the treatment of local adaptation syndrome]. Dermatologia Ambulatoriale. 2007;2:29-35. Italian.
Clinical, Cosmetic and Investigational Dermatology

\section{Publish your work in this journal}

Clinical, Cosmetic and Investigational Dermatology is an international, peer-reviewed, open access, online journal that focuses on the latest clinical and experimental research in all aspects of skin disease and cosmetic interventions. All areas of dermatology will be covered; contributions will be welcomed from all clinicians and

\section{Dovepress}

basic science researchers globally. This journal is indexed on CAS The manuscript management system is completely online and includes a very quick and fair peer-review system, which is all easy to use. Visit http://www.dovepress.com/testimonials.php to read real quotes from published authors. 